\title{
INVESTIGATION OF ERGONOMIC RISK FACTORS AMONG MALE WORKERS IN A MEDICAL MANUFACTURING COMPANY IN NORTHERN MALAYSIA
}

\author{
Athirah Yusof and Nurul Shahida Mohd Shalahim \\ Faculty of Mechanical Engineering, University Malaysia Pahang, Pekan, Pahang, Malaysia
}

*Corresponding author: Athirah Yusof

Email address: athirah_yusof@ymail.com

\begin{abstract}
The medical manufacturing industry is currently one of the sectors with great potential growth in Malaysia, which offers numerous job opportunities to local and foreign workers. The growing proportion of workers in this industry means that a large number of workers are potentially exposed to ergonomic risk factors at the workplace, which may consequently affect their health. Thus, this study was carried out is to investigate the ergonomic risk factors and the prevalence of musculoskeletal disorders among male workers in a medical manufacturing company in northern Malaysia. Eight industrial workers $(n=8)$ were recruited from two Plastic Technology (PT) departments to participate in this study. Observations were made by recording the participants performing two tasks (manual lifting of containers and bags of chemicals) using a video camera at the PT1 and PT3 departments. The participants' personal information and their job-related characteristics were first collected using a demographic questionnaire. Next, the Cornell Musculoskeletal and Hand Discomfort Questionnaire (CMDQ) was used to evaluate the prevalence of musculoskeletal disorders among workers while Manual Handling Assessment Charts (MAC) were used to assess the most common risk factors during the lifting operations. The results showed that the highest prevalence of musculoskeletal disorders among the male workers at the medical manufacturing company was at the lower back (83.17\%), upper back (4.38\%), right shoulder (3.49\%), and left shoulder (3.49\%). Based on the results of the MAC, $100 \%$ of the participants were at high risk of injury from postures in the vertical lift zones, torso twisting, and sideways bending. In addition, 87.5 and 62.5\% of the participants were at high risk of injury based on their hand distance from the lower back and grip on the load, respectively. The results of this study can serve as a guideline to implement interventional ergonomics programmes at the workplace and improve the musculoskeletal health of workers in the medical manufacturing industry in Malaysia.
\end{abstract}

Keywords: Ergonomic assessment, ergonomic risk factors, manual material handling, manufacturing industry

\section{INTRODUCTION}

The manufacturing industry plays a vital role in transforming the economy landscape in Malaysia. According to the Department of Statistics Malaysia, Malaysia's manufacturing sales increased to MYR 72.4 billion in March 2019 from MYR 68.5 billion for the same month in the preceding year, which corresponds to an increase of $5.7 \%{ }^{1}$. Even though the manufacturing industry is diverse in Malaysia, the sales are significant in the following sectors: (1) food, beverages, and tobacco, (2) petroleum, chemicals, rubbers, and plastics, (3) textile, apparel, leather, and footwear, (4) non-metallic mineral products, (5) basic metals and fabricated metal products, (6) wood, furniture, paper products, and painting, (7) electrical and electronic products, and (8) transport equipment and other types of manufacturing products.

In order to improve safety and boost productivity, machinery and equipment are used in place of human workers in heavy industries. Nonetheless, in the manufacturing industry, human workers are still required to perform manual handling tasks ${ }^{2}$ involving manual lifting, lowering, carrying, pushing, and pulling objects or materials $3-5$. Manual handling tasks can be stressful to the body, which can lead to musculoskeletal disorders
(MSDs). MSDs refer to injuries that affect the muscles, nerves, joints, ligaments, tendons, or spinal disc owing to the nature of the work ${ }^{3,6}$. MSDs are a common health problem not only in the manufacturing industry, but also in various sectors around the world ${ }^{6-9}$. MSDs will significantly affect the productivity, absenteeism, compensation, turnover, and quality of work of workers ${ }^{10}$.

According to the statistics published by the Department of Occupational Safety and Health, Malaysia, the manufacturing sector ranks first for occupational accidents until October $2018{ }^{11}$. The rising workforce and work demand increase the risk of workers to be exposed to occupational accidents and diseases. Performing jobs in an awkward posture, handling heavy loads, working in extreme temperatures, performing highly repetitive tasks, working in a static and sustained work posture, and regular exposure to excessive noise are the major ergonomic risk factors that contribute to occupational accidents and diseases $8,12,13$. Hence, this study was carried out to (1) assess the prevalence of MSDs among male workers in a medical manufacturing company and (2) determine the level of ergonomic risk factors among the male workers in the medical manufacturing company. 


\section{STUDY DESIGN AND METHODS}

This study was conducted in a medical manufacturing company located at Bayan Lepas, Penang, over a three-month period. This company consists of a few administrative offices and several production departments, which fabricate medical equipment for infusion therapy, as well as manufacture pharmaceutical solutions and surgical instruments for patient care in Malaysia and around the world. Eight material handlers were recruited from two Plastic Technology (PT) departments to participate in this study. The tasks chosen for this investigation were manual handling of (1) chemical bags and (2) tumbling containers (Figure 1), which involve lifting, twisting, lowering, and repetitive motions. The participants were briefed regarding the purpose and methodology of the study and written consent was obtained from each participant. Cash vouchers were given to the participants as a token of appreciation for participating in this study.

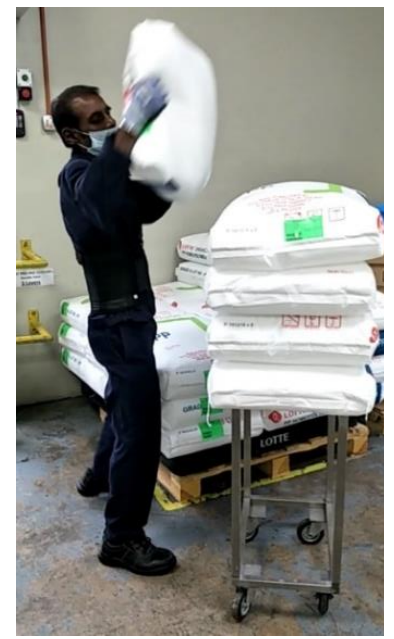

(a)

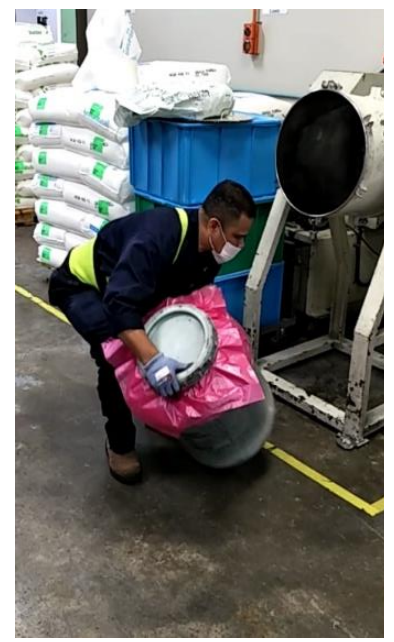

(b)
Figure 1: Manual lifting of a (a) $25-\mathrm{kg}$ chemical bag and (b) 28.61-kg tumbling container

Screening was first carried out by conducting a walkthrough at the PT departments. After the screening process, two tasks were chosen (one for PT1 and PT3, respectively) based on the ergonomic hazards and risk factors identified. The tasks were recorded by using a video camera so that they could be paused or viewed again if necessary. In addition, the video recordings enable the working conditions such as body posture to be examined clearly and any missing information could be retrieved at any time.

Two data collection methods were used in this study: (1) self-assessment using a demographic questionnaire and the Cornell Musculoskeletal Disorder Questionnaire (CMDQ), and (2) direct observations of the workers using the Manual Handling Assessment Charts (MAC). These assessment tools were conducted in accordance with the Guidelines on Ergonomics Risk Assessment at Workplace 2017 by the Department of Occupational Safety and Health, Malaysia ${ }^{14}$. Once the assessment was done and the ergonomic risk factors were identified, the appropriate control measures were proposed to minimize occupational risk of injuries. Figure 2 shows the process flow of this study.

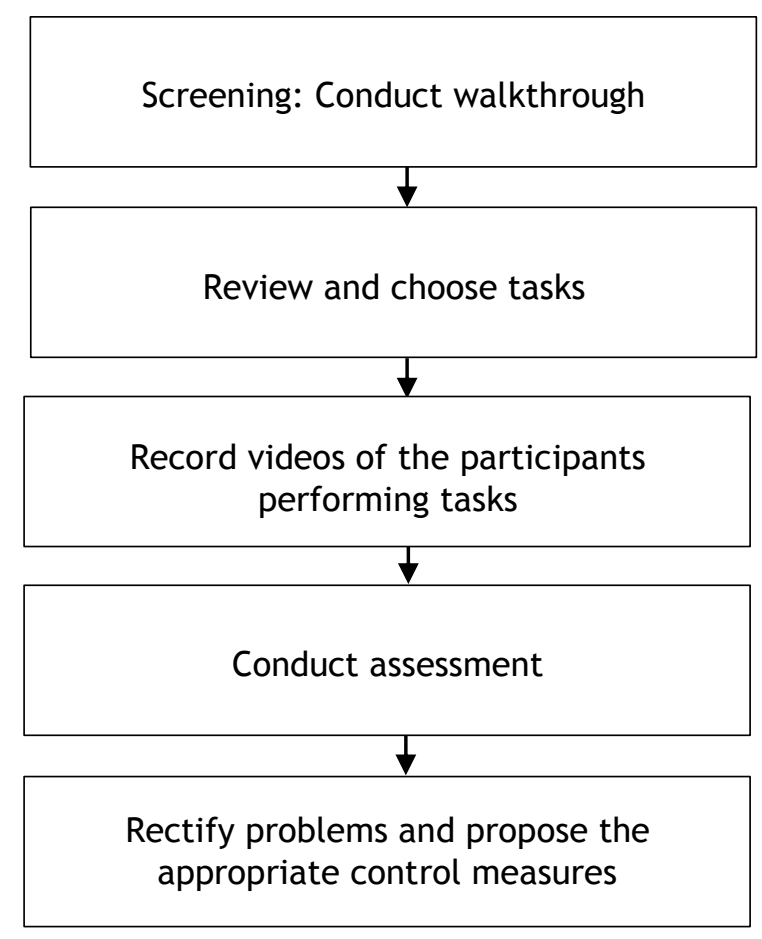

Figure 2: Process flow of this study

\section{DATA COLLECTION}

\section{Demographic questionnaire}

The demographic questionnaire was distributed to the operators and material handlers at the time of entry to the study to obtain their personal information and job-related characteristics. The questionnaire consisted of four parts: (a) sociodemographic background, (b) working conditions, (c) social/lifestyle, and (d) medical history. Part (a) was used to obtain general information of each participant: name, employee number, age, gender, height, weight, race, highest educational level, and marital status. Part (b) was used to obtain information on each participant's nature of work: department, job title, type of employment (full-time, part-time, or shift work), number of breaks, break duration, and the number of people working with them. The social characteristics/lifestyle habits of the participants were identified in Part (c), where the questions involved determining whether the participants smoked or not, the number of cigarettes smoked per day if the participants were smokers, and their hobby during their leisure time. Part (d) was used to elicit information on the medical history 
of the participants, where the questions involved determining whether the participants were diagnosed of any diseases by doctors and whether they were taking any medications. The questionnaire was provided with checkboxes to assist the participants in answering each question accordingly. The participants were requested to complete the demographic questionnaire before performing the tasks while being fully monitored by the researchers.

\section{CORNELL MUSCULOSKELETAL DISORDER QUESTIONNAIRE}

The CMDQ consisted of 54 items for 18 regions of the body parts along with a body-map diagram.
The CMDQ was distributed to the participants on their last working day of the week. The 18 regions of the body parts included the neck, shoulders, upper back, upper arms, lower back, forearms, wrists, hip/buttocks, thighs, knees, lower legs, and feet. The CMDQ comprised three parts (frequencies, discomfort, and interference) and the rating scale for each part is shown in Table 1. According to the CMDQ scoring guidelines ${ }^{15}$, the total discomfort score can be determined based on the scores for the frequency, discomfort, and interference, as follows:

Total discomfort score $=$ Frequency score $x$ Discomfort score $\times$ Interference score

\section{Table 1: Rating score for frequency, discomfort, and interference}

\begin{tabular}{ccc}
\hline Frequency & Discomfort & Interference \\
\hline Never $=0.0$ & Slightly uncomfortable $=1$ & Not at all $=1$ \\
$1-2$ times $/$ week $=1.5$ & Moderately uncomfortable $=2$ & Slightly interfered $=2$ \\
$3-4$ times $/$ week $=3.5$ & Very uncomfortable $=3$ & Substantially interfered $=3$ \\
Every day $=5.0$ & & \\
Several times every day $=10.0$ & & \\
\hline
\end{tabular}

\section{Manual Handling Assessment Charts}

In this study, the common risk factors of the lifting operations were assessed by using the MAC. The MAC were developed by the Health and Safety Executive UK ${ }^{16}$ to assist employers, health and safety managers, and safety representatives in identifying high-risk manual handling tasks (including lifting and carrying) and team handling operations. Based on the video recordings, the level of each risk factor was determined by observing the task based on the assessment guide and flow chart. The colour bands (green, amber, red, and purple) and the corresponding numerical scores were entered into the MAC score sheet and the total score was determined. These colour bands facilitate in determining elements of the task that require attention while the scores assist in prioritizing the tasks that require the most urgent attention.

\section{RESULTS}

\section{Outcomes of the demographic questionnaire}

The eight participants involved in this study were found to be 41-55 years old (mean $=48.75, \mathrm{SD}=$ 4.65). From the total number of participants, five participants $(62.5 \%)$ were Malays while the rest
$(37.5 \%)$ were Indians. Most of the participants $(87.5 \%)$ received education up to secondary level and all of them (100.0\%) were married. Five participants $(62.5 \%)$ had a working experience of less than 10 years, one participant $(12.5 \%)$ had less than 20 years of experience, and two participants $(25.0 \%)$ had more than 20 years of working experience. For working conditions, three participants (37.5\%) were from PT1 black area whereas the remaining participants $(62.5 \%)$ were from the PT3 black area. All participants (100.0\%) worked as material handlers. Seven participants $(87.5 \%)$ were found to work in shifts. Three participants $(37.5 \%)$ were entitled to one break during the day, and only one participant (12.5\%) was entitled to two breaks. There were two periods of break: $60 \mathrm{~min}$ and $70 \mathrm{~min}$. Four participants $(50 \%)$ had a break period of $60 \mathrm{~min}$ whereas the remaining half had a break period of $70 \mathrm{~min}$. As for their social/lifestyle, five participants $(62.5 \%)$ were non-smokers. Four participants (50\%) chose jogging as their hobby, two participants (25\%) loved to play football, whereas the remaining participants chose other activities as their hobby. Lastly, in terms of the medical history, four participants (50\%) were diagnosed with disease by doctors and they were taking medications. The results obtained from the demographic questionnaire are summarized in Table 2. 
Table 2: Demographic information of the participants in this study

\begin{tabular}{lcc}
\hline Demographic criteria & $\begin{array}{c}\text { Number of } \\
\text { participants }\end{array}$ & $\begin{array}{c}\text { Percentage of } \\
\text { participants (\%) }\end{array}$ \\
\hline
\end{tabular}

A) Socio-demographic background

Age

$41-45$ years
$46-50$ years

25.0

25.0

Race

Malay

Indian

Highest educational level

Primary

Secondary

Marital status

Married

Single

$\begin{array}{lr}2 & 25.0 \\ 2 & 25.0 \\ 4 & 50.0 \\ 5 & \\ 3 & 62.5 \\ & 37.5 \\ 7 & \\ 7 & 12.5 \\ 8 & 87.5 \\ 0 & \\ & \\ 5 & 00.0 \\ 1 & \\ 2 & 62.5 \\ & 12.5 \\ & 25.0\end{array}$

B) Working conditions

Department

PT1 black area

PT3 black area

37.5

Job title

Material handler

62.5

Type of employment

Full-time

Shift work

62.5

37.5

12.5

87.5

00.0

62.5

12.5
25.0

Number of breaks

Once

Twice

3

5

100.0

Break period

$60 \mathrm{~min}$

12.5

70 min

C) Social characteristics/lifestyle habits

Smoker

Yes

No

Hobby

Jogging

Football

Others

$\begin{array}{lc}3 & 37.5 \\ 5 & 62.5 \\ & \\ 4 & 50 \\ 2 & 25 \\ 2 & 25\end{array}$

D) Medical history

Diagnosed of any diseases?

Yes

No

$4 \quad 50$

Taking any medications?

Yes

$4 \quad 50$

No

$4 \quad 50$

450

\section{OUTCOMES OF THE CORNELL MUSCULOSKELETAL DISORDER QUESTIONNAIRE}

Table 3 shows the total discomfort scores and the corresponding rank for each body part. Most of the participants experienced discomfort at the lower back $(83.17 \%)$, followed by the upper back (4.38\%), and shoulders (right: $3.49 \%$, left: $3.49 \%$ ).
Most of the them felt more discomfort at the upper body parts (neck, shoulders, upper back, upper arms, lower back, forearms, and wrists) than the lower body parts (hip/buttocks, thighs, knees, lower legs, and feet). This is likely because the upper body parts experience more stress during the lifting and lowering tasks. Among the upper body parts, the discomfort was more severe at the neck $(0.68 \%)$ and left forearm $(0.68 \%)$ than 
the upper arms (right: $0.16 \%$, left: $0.02 \%$ ). Meanwhile, the total discomfort scores were $0.00 \%$ for the right forearm and both wrists, indicating that the discomfort experienced by the participants was negligible and therefore, these body parts were ranked the lowest among all body parts. For lower body parts, the discomfort scores for the left foot (1.85\%) and thighs (right: 0.91\%, left: $0.91 \%)$ indicated that the discomfort was more severe in these body parts compared with that in the left knee $(0.11 \%)$, lower left leg
$(0.07 \%)$, and right foot $(0.01 \%)$. In contrast, the total discomfort scores were $0.00 \%$ for three lower body parts (hip/buttocks, right knee, and lower right leg) because there were no discomfort scores recorded. Based on the CMDQ results, it can be deduced that the lower back and upper back were the most critical body parts whereas the right forearm, wrists, right knee, and lower right leg were the least critical body parts in which discomfort was experienced during manual lifting and lowering operations.

Table 3: Total discomfort score and corresponding rank for each body part

\begin{tabular}{|c|c|c|c|c|c|c|}
\hline Body part & $\begin{array}{l}\text { Frequency } \\
\text { score }\end{array}$ & $\begin{array}{l}\text { Discomfort } \\
\text { score }\end{array}$ & $\begin{array}{l}\text { Interference } \\
\text { score }\end{array}$ & $\begin{array}{c}\text { Total } \\
\text { discomfort } \\
\text { score }\end{array}$ & $\begin{array}{c}\text { Total } \\
\text { discomfort } \\
\text { score (\%) }\end{array}$ & Rank \\
\hline Neck & 3.0 & 5 & 4 & 60.0 & 0.68 & 8 \\
\hline \multirow[t]{2}{*}{ Shoulder } & 8.5 & 6 & 6 & 306.0 & 3.49 & 3 \\
\hline & 8.5 & 6 & 6 & 306.0 & 3.49 & 3 \\
\hline \multirow{2}{*}{$\begin{array}{l}\text { Upper back } \\
\text { Upper arm (Right) }\end{array}$} & 8.0 & 8 & 6 & 384.0 & 4.38 & 2 \\
\hline & 3.5 & 2 & 2 & 14.0 & 0.16 & 10 \\
\hline (Left) & 1.5 & 1 & 1 & 1.5 & 0.02 & 14 \\
\hline Lower back & 28.5 & 16 & 16 & 7296.0 & 83.17 & 1 \\
\hline Forearm & 0.0 & 0 & 0 & 0.0 & 0.00 & 15 \\
\hline (Left) & 5.0 & 3 & 4 & 60.0 & 0.68 & 8 \\
\hline \multirow[t]{2}{*}{ Wrist } & 0.0 & 0 & 0 & 0.0 & 0.00 & 15 \\
\hline & 0.0 & 0 & 0 & 0.0 & 0.00 & 15 \\
\hline Hip/buttocks & 0.0 & 0 & 0 & 0.0 & 0.00 & 15 \\
\hline \multirow[t]{2}{*}{ Thigh } & 5.0 & 4 & 4 & 80.0 & 0.91 & 6 \\
\hline & 5.0 & 4 & 4 & 80.0 & 0.91 & 6 \\
\hline \multirow[t]{2}{*}{ Knee } & 0.0 & 0 & 0 & 0.0 & 0.00 & 15 \\
\hline & 5.0 & 2 & 1 & 10.0 & 0.11 & 11 \\
\hline \multirow[t]{2}{*}{ Lower leg } & 0.0 & 0 & 0 & 0.0 & 0.00 & 15 \\
\hline & 1.5 & 2 & 2 & 6.0 & 0.07 & 12 \\
\hline \multirow[t]{2}{*}{ Foot } & 1.5 & 2 & 2 & 6.0 & 0.07 & 12 \\
\hline & 6.5 & 5 & 5 & 162.5 & 1.85 & 5 \\
\hline
\end{tabular}

\section{OUTCOMES OF THE MAC}

Figure 5 shows the levels of risk for the ergonomic risk factors. Based on the MAC assessment, all participants $(100 \%)$ were at medium risk of injury in terms of the load weight/frequency. The majority of participants (87.5\%) were at high risk of injury whereas $12.5 \%$ were at medium risk of injury based on their hand distance from the lower back risk. All participants (100\%) were at high risk of injury from postures in the vertical lift zones, torso twisting, and sideways bending. In addition,
$62.5 \%$ of the participants were at high risk whereas $37.5 \%$ were at medium risk because of their grip on load. All participants $(100.0 \%)$ were at low risk of injury because of factors that modify their postures (postural constraints), floor conditions where the manual handling tasks are carried out (floor surface), and environmental factors. Overall, the level of risk of injury was high (as indicated by the red band in Figure 3) for all participants and the corresponding MAC score was within a range of $13-20$, indicating that action is required soon. 


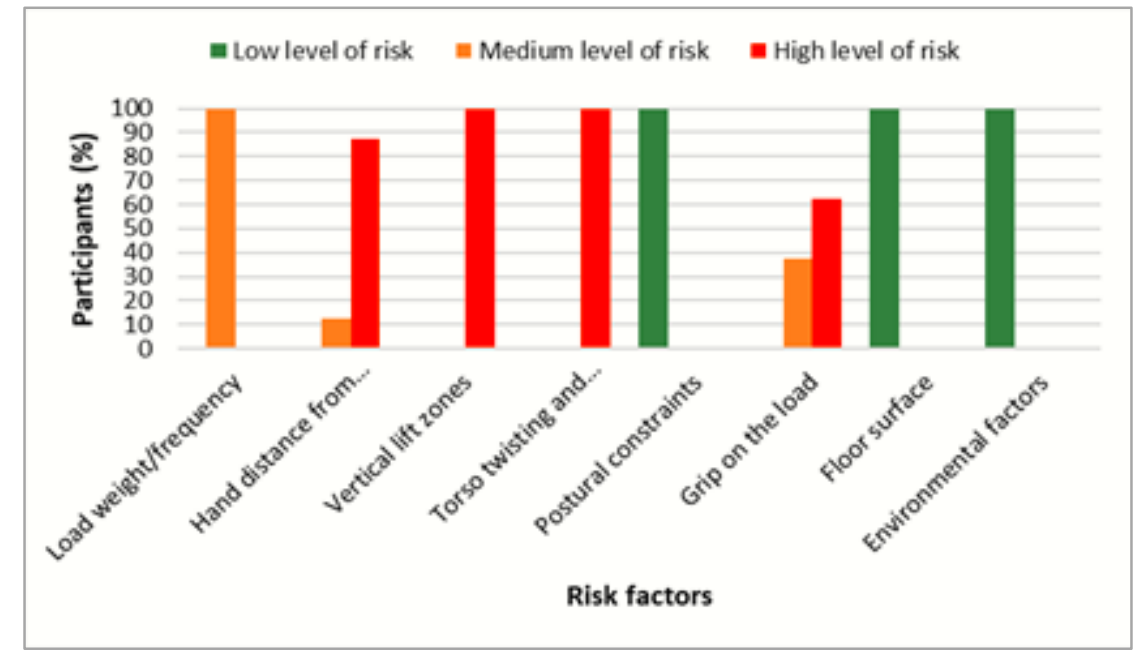

Figure 3: Levels of risk for all ergonomic risk factors

\section{DISCUSSION}

This study explores the prevalence of MSDs and the levels of risk for ergonomic risk factors among male workers in a medical manufacturing company in Malaysia. The results showed that the material handlers who performed manual lifting and lowering tasks experienced symptoms of MSDs and ergonomic risks owing to awkward and extreme postures. The CMDQ results indicated that MSDs were most prevalent at the lower back $(83.17 \%)$, followed by the upper back $(4.38 \%)$, and right shoulder (3.49\%). The results were consistent with those of Aziz et al. ${ }^{17}$, Mean et al. ${ }^{18}$, and Soltanzadeh et al. ${ }^{19}$, who found that MSDs were most prevalent at the lower back.

However, there were also studies that showed different results. For example, Daruis et al. ${ }^{2}$ and Lu et al. ${ }^{12}$ discovered that MSDs were most prevalent at the shoulders and neck, respectively. Meanwhile, Zare et al. ${ }^{7}$ conducted a study among truck assembly plant workers and the results indicated that the highest prevalence of MSDs was at the shoulders and elbows. In addition, most of the workers felt more discomfort at the upper body parts than at the lower body parts because the upper body parts experienced more stress during lifting and lowering operations.

Performing manual handling tasks in a production facility is demanding and requires a high degree of strength to cope with the heavy loads. In this study, the risk levels of MSDs among material handlers were analysed to identify if the manual handling tasks would lead to a high risk of injury among the male workers in the medical manufacturing company. The MAC assessment results indicated that all participants $(100.0 \%)$ were at high risk of injury for postures in the vertical lift zones, torso twisting, and sideways bending, $87.5 \%$ were at high risk of injury because of their hand distance from the lower back, while
$62.5 \%$ were at high risk of injury owing to their grip on the load. In general, the level of risk of injury was high for all participants (100.0\%), where the corresponding MAC score was within a range of 13-20. This indicates that control measures should be implemented in the near future for the manual handling tasks investigated in this study in order to reduce the risk of musculoskeletal injuries. The results of this study differ from those of Soltanzadeh et al. ${ }^{19}$ whose focus was on the workers in the rubber industry, where the level of risk was in the unacceptable range for $46.3 \%$ of their participants, as indicated by the purple colour band in Figure 3 . In such a case, immediate control measures need to be implemented.

\section{CONCLUSION}

The prevalence, discomfort, and interference of MSDs and the levels of ergonomic risk factors of male workers in a medical manufacturing company in northern Malaysia have been investigated in this study. The results showed that the pain experienced by the workers at the lower back, upper back, shoulders, left foot, and thighs was higher compared with that at other body parts. In addition, the results showed that the level of risk of injury was high for the participants because of postures in the vertical lift zones, torso twisting, and sideways bending, and because of their hand distance from lower back. Based on the MAC results, it can be concluded that the tasks conducted by all material handlers were in the high level of risk of injury. Hence, it is proposed that development and intervention programmes such as awareness training and education should be cultivated to reduce the level for risk among the material handlers. In addition, it is recommended to reduce the load weight, improve the rest time, and improve personal protective equipment. 


\section{ACKNOWLEDGEMENTS}

The authors acknowledge the Ministry of Higher Education Malaysia (MoHE) and Universiti Malaysia Pahang (UMP) for funding this work (Grant No.: RDU180388).

\section{REFERENCES}

1. Department of Statistics Malaysia OP. Manufacturing Statistics 2019 [Available from:

https://www.dosm.gov.my/v1/index.ph $\mathrm{p} ? \mathrm{r}=$ column $/$ cthemeByCat\&cat $=90 \& b u l \_i d$ $=$ N091MGlpVIJxQ2k2dUZ1OFNhT21JZz09\& menu_id=SjgwNXdiMOJIT3Q2TDBIWXdKdU Vldz09.

2. Daruis D, Liyana Rosly A, Abd Aziz I, Syazwani Hishamuddin N, Deros B. Ergonomic risk assessment of manual material handling at an automotive manufacturing company2017. 317-24 p.

3. Santos A, Ramos HM, Ramasamy G, Fernandes C. Prevalence of musculoskeletal pain in a sample of migrant workers in the manufacturing industry in Malaysia. Public Health. 2014;128(8):759-61.

4. Deros BM, Daruis DDI, Basir IM. A Study on Ergonomic Awareness among Workers Performing Manual Material Handling Activities. Procedia - Social and Behavioral Sciences. 2015;195:1666-73.

5. Valentina V, Fabio S, Martina C, Alessandro P. Fatigue accumulation in the assignment of manual material handling activities to operators. IFACPapersOnLine. 2018;51(11):826-31.

6. Nurmianto E, Ciptomulyono U, Suparno, Kromodihardjo S. Manual Handling Problem Identification in Mining Industry: An Ergonomic Perspective. Procedia Manufacturing. 2015;4:89-97.

7. Zare $M$, Malinge-Oudenot A, Höglund $R$, Biau S, Roquelaure $Y$. Evaluation of ergonomic physical risk factors in a truck manufacturing plant: case study in SCANIA Production Angers. Industrial Health. 2016;54(2):163-76.

8. Mork MA, Choi SD. An ergonomic assessment of sample preparation job tasks in a chemical laboratory. Journal of Chemical Health and Safety. 2015;22(4):23-32.
9. Garosi E, Mazloumi A, Kalantari R, Vahedi $Z$, Shirzhiyan Z. Design and ergonomic assessment of an infusion set connector tool used in nursing work. Applied Ergonomics. 2019;75:91-8.

10. S Hebbal S, C S Kumar A, S. M Q. An ergonomic study of work related musculoskeletal disorder risks in Indian Saw Mills2014.

11. Department of Occupational Safety and Health Ministry of Human Resources Malaysia. Occupational Accident Statistics by Sector 2018 [Available from: http://www.dosh.gov.my/index.php/en/ occupational-accident-statistics/bysector.

12. Lu J-M, Twu L-J, Wang M-JJ. Risk assessments of work-related musculoskeletal disorders among the TFTLCD manufacturing operators. International Journal of Industrial Ergonomics. 2016;52:40-51.

13. Asadi H, Yu D, Mott JH. Risk factors for musculoskeletal injuries in airline maintenance, repair \& overhaul. International Journal of Industrial Ergonomics. 2019;70:107-15.

14. Department of Occupational Safety and Health Ministry of Human Resources Malaysia. Guidelines On Ergonomics Risk Assessment At Workplace 20172017 [Available from: http://www.dosh.gov.my/index.php/en/ legislation/guidelines/ergonomic/2621-

01 -guidelines-on-ergonomics-riskassessment-at-workplace-2017/file.

15. Cornell University Ergonomics Web. Cornell Musculoskeletal Discomfort Questionnaires (CMDQ) 2003 [Available from:

http://www.ergo.human.cornell.edu/ah msquest.html.

16. Health and Safety Executive. Manual handling assessment chart 2003 [Available from:

http://www.hse.gov.uk/msd/mac/index. htm.

17. Aziz FA, Ghazalli Z, Mohamed NMZ, Isfar A, lop. Investigation on musculoskeletal discomfort and ergonomics risk factors among production team members at an automotive component assembly plant. 4th International Conference on Mechanical Engineering Research. IOP 
Conference Series-Materials Science and Engineering. 257. Bristol: lop Publishing Ltd; 2017.

18. Mean V, Abdullah N, Dawal S, Aoyama H, Sothea K. Investigation on Musculoskeletal Symptoms and Ergonomic Risk Factors at Metal Stamping Industry2013. 293-9 p.

19. Soltanzadeh A, Abedini R, Choobineh A, Gholami M, Amiri F, Almasi Hashyani A. Ergonomic Risk Assessment of Lifting Activities; a Case Study in a Rubber Industry. Jundishapur Scientific Medical Journal. 2013;5(1):9-15. 
